\title{
An ECG signal model based on a parametric description of the characteristic waves
}

\author{
Pavol Dolinský1, Imrich Andrášs ${ }^{1}$, Linus Michaeli ${ }^{1}$, Ján Šaliga ${ }^{1}$ \\ ${ }^{1}$ Department of Electronics and Multimedia Telecommunications, Faculty of Electrical Engineering and Informatics, Technical University of \\ Košice, Letná 9, 04200 Košice, Slovakia
}

\begin{abstract}
This article introduces a new electrocardiogram (ECG) signal model based on geometric signal properties. Instead of the artificial functions used in common ECG models, the proposed model is based on the modelling of real ECG signals divided into time segments. Each segment has been modelled using simple geometrical forms. The final ECG signal model is represented by the sequence of parameters of the base functions. Parameter variations allow for the generation of different waveforms for each subsequent heartbeat without mixing up the PQRST waves order. Two basic models utilize slightly modified elementary functions, which are computationally simple. A combination of both models allows for the modelling of irregularities in the consecutive heartbeats of the specific ECG waveforms. Respiratory, noise, and powerline interference can be added in order to make the generated ECG signal more realistic. The model parameters are estimated by differential evolution optimization and a comparison between the modelled ECG and the acquired signal. The proposed models are tested by the database included in the LabVIEW Biomedical Toolkit and ECG records in the MIT-BIH arrhythmia database.
\end{abstract}

\section{Section: RESEARCH PAPER}

Keywords: Synthetic ECG; ECG signal processing; ECG model; PQRST complex

Citation: Pavol Dolinský, Imrich Andráš, Linus Michaeli, Ján Šaliga, An ECG signal model based on a parametric description of the characteristic waves, Acta IMEKO, vol. 9, no. 2, article 2, June 2020, identifier: IMEKO-ACTA-09 (2020)-02-02

Editor: Alexandru Salceanu, Technical University of lasi, Romania

Received December 9, 2019; In final form April 9, 2020; Published June 2020

Copyright: This is an open-access article distributed under the terms of the Creative Commons Attribution 3.0 License, which permits unrestricted use, distribution, and reproduction in any medium, provided the original author and source are credited.

Corresponding author: Ján Šaliga, e-mail: jan.saliga@tuke.sk

\section{INTRODUCTION}

Mathematical modelling allows for a description - by mathematical tools - of the properties of complex mechanical, electric, and any systems in which the final behaviours result from the physical relationships of various origins acting in a complex manner. These include behaviour analysis and the prediction of electronic [1], mechanical [2]-[4], biological [5] system components, thermal systems [6][7], data transmission channels [8], and more. A mathematical model gives concise information about basic processes, without the effects of low significance. Such modelling is beneficial in that its accuracy can be controlled according to user needs. Electrical modelling is especially suitable for biomedical systems, where the noise and several inherent artefacts influence the measured signal from the output of the observed biological object. The best examples of applying mathematical modelling of biological signals is the evaluation and extraction of electrocardiogram (ECG) signals [9]-[11] and compressed sensing [12]-[14][16].
The human heart generates spatio-temporal electric potential during contractions in the cardiac pump cycle. The measured potential propagates through human tissues on the skin surface. The difference of electric potential between electrodes placed on the selected positions of the body is acquired and converted to a standardized ECG. Because ECG is a result of the propagation through the human body, it contains artefacts caused by muscle and respiration movement; powerline and other external noise sources; and spurious distortion from non-perfect electrode contacts [15].

Databases of real recordings, such as Phisionet [17], are often used for model evaluation, but it may be difficult to assess how a particular method reacts in the case of the same basic signal, with different levels of noise and artefacts. An alternative approach is to generate synthetic biomedical signals by using the suitable signal model and comparing its output signal with the measured one.

In the past, a wide variety of different ECG signal models have been developed and tested. One of the most basic ECG models was published in [18]. In [19], the rational Bézier curves 
and Bernstein polynomials were used to model heartbeat curves. The Gaussian curve is also a very common method of modelling ECG signal. An evaluation and comparison concerning mathematical models for the extraction of QRS features was done in [21]. Gaussian, Mexican hat, and Rayleigh functions were used for the whole heartbeat period of the modelling. Adaptive weighted Hermit functions were used in [22]. An original, alternative method was introduced in [23], where a QRS wave group was modelled using the Ant Colony algorithm. The researchers in [24] simulated real-time ECG waveforms by means of the superposition of bounded functions. This property is a defining feature of most periodic functions. An alternative method of the simulation of ECG signals the spectrum-based one [25]. Among the well-known, most widely used ECG signal models, the dynamic model [26] is based on three coupled differential equations.

The approach presented in this paper is significantly different to all of these. It is based on the elementary trigonometric and linear functions or the derivation of the Gaussian pulse to model each individual wave. Computational simplicity is the main advantage of these models, which allows for the implementation on embedded devices thereof for denoising, automated patient diagnostics, etc.

\section{ECG SIGNAL}

The ECG signal is described by five consecutive waves, labelled with the letters $\mathrm{P}, \mathrm{Q}, \mathrm{R}, \mathrm{S}$, and T, as is standard (Figure 1). These waves correspond to the depolarization and repolarization of heart muscles [26] during one heartbeat.

The initial $\mathrm{P}$ wave represents the activation of atria and is followed by a relatively short isoelectric segment, while the cardiac impulse passes through the $\mathrm{A}-\mathrm{V}$ node and the HisPurkinje system. The excitation and muscle contraction represent the main force behind blood circulation to the organs and creates the tallest QRS complex. It has three components. The first downward deflection is called the $\mathrm{Q}$ wave, the consecutive upward deflection is called the $\mathrm{R}$ wave, and the final downward deflection is called the $\mathrm{S}$ wave. The polarity of each wave in the QRS complex may vary according to the position of sensing leads as well as body abnormalities. A normal ECG beat ends with a low-frequency $\mathrm{T}$ wave, during which the ventricles return to their electrical resting state.

\section{THE PROPOSED ECG SIGNAL MODEL}

The authors initially proposed two signal models, in which each wave of a PQRST complex is modelled by elementary concatenated mathematical functions. The models differ in the modelling of the $\mathrm{Q}$ and $\mathrm{S}$ waves. The first one uses the fixed shape of $\mathrm{Q}$ and $\mathrm{S}$ waves with the adjustable time of origin, width,

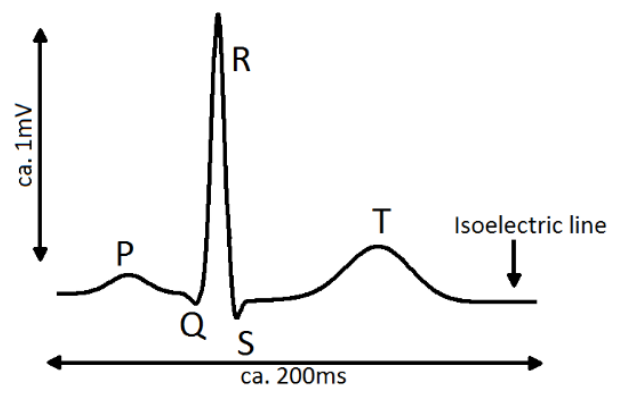

Figure 1. PQRST complex of a normal lead II ECG recording. and amplitude. The second ECG signal model additionally allows for the adaptation even of their shapes.

Finally, the third custom ECG signal model is based on linking the cardiac cycles of the first two models, where the vectors of their parameters may differ in each PQRST complex. It allows for the generation of a model of a different waveform for each subsequent heartbeat or the superposition of different heart rate disturbances at predefined positions.

These models can include the interference of Gaussian and powerline noise as well as interference caused by respiration.

\subsection{Fixed-shape $\mathrm{Q}$ and $\mathrm{S}$ waves}

The heartbeat starts with a short isoelectric segment. It is modelled by the simple constant:

$B(k)=0$; for $0 \leq k \leq K_{B}$,

where $K_{B}$ is the width and $k$ is the sample number within one wave segment. The consecutive $\mathrm{P}$ wave is modelled by the cosine function:

$P(k)=-A_{P} \cos \left(\frac{2 \pi k+15}{K_{P}}\right) ;$ for $0 \leq k \leq K_{P}$,

where $A_{P}$ is the amplitude and $K_{P}$ is the width of the $\mathrm{P}$ wave. $\mathrm{A}$ short isoelectric segment follows:

$P_{Q}(k)=0 ;$ for $0<k \leq K_{P Q}$,

where $K_{P Q}$ is the width. The Q wave is modelled using a segment created by a section of a Gaussian monopulse (a differentiated Gaussian pulse):

$Q(k)=\frac{A_{Q}\left(\mathrm{k}-0.1 \mathrm{~K}_{\mathrm{Q}}+0.1\right) 19.78 \pi}{K_{Q}} e^{-2\left(\frac{6 \pi}{K_{Q}}\left(\mathrm{k}-0.1 K_{Q}+0.1\right)\right)^{2}}$

for $0 \leq k \leq K_{Q}$, where $A_{Q}$ is the amplitude and $K_{Q}$ is the width of $\mathrm{Q}$ wave. The $\mathrm{R}$ wave is modelled using a sinewave segment:

$R(k)=A_{R} \sin \left(\frac{\pi k}{K_{R}}\right) ;$ for $0 \leq k \leq K_{R}$,

where $A_{R}$ is the amplitude and $K_{R}$ is the width of $\mathrm{R}$ wave. The $\mathrm{S}$ wave is modelled by a segment of a Gaussian monopulse:

$S(k)=-A_{S} 0.1 k \frac{19.78 \pi}{K_{S}} e^{-2\left(\frac{6 \pi}{K_{S}} 0.1 k\right)^{2}}$ for $0 \leq k \leq K_{S}-K_{C S}$,

where parameter $A_{S}$ is the amplitude, and $K_{S}$ is the basic width of the $\mathrm{S}$ wave. $K_{C S}$ is a parameter that allows for the slight adjustment of the $\mathrm{S}$ wave shape by cutting away a portion at the end. The transition between the $\mathrm{S}$ and $\mathrm{T}$ wave is described as a linear function:

$S_{T}(k)=-S\left(K_{S}-K_{C S}\right) \frac{k}{s_{m}}+S\left(K_{S}-K_{C S}\right)$

for $0 \leq k \leq K_{S T}$, where $s_{m}$ is the slope parameter, and $K_{S T}$ is the width of the transition segment. The $T$ wave is modelled using a segment of the cosine function:

$T(k)=-A_{T} \cos \left(\frac{1.48 \pi \mathrm{k}+15}{K_{T}}\right)+A_{T}+S_{T}\left(K_{S T}\right)$ 
for $0 \leq k \leq K_{T}$, where $A_{T}$ is the amplitude and $K_{T}$ is the width of the $\mathrm{T}$ wave. The final transition from the $\mathrm{T}$ wave back to the isoelectric line is modelled using the function:

$I(k)=T\left(K_{T}\right) \frac{s_{I}}{k+10} ;$ for $0 \leq k \leq K_{I}$,

where $s_{I}$ is the parameter for setting the transition slope between the T wave and isoelectric line, and $K_{I}$ is the width of the ending section.

The concatenation of all modelled $H\left({ }^{1} b, n\right)$ (Equations 1-8) describes the complete heartbeat. The beginning of each segment is appended to the end of a previous one, while their order is always the same starting with Equation (1) and ending with Equation (8). Here, parameter ${ }^{1} b$ denotes the set of heartbeat parameters for the first variant:

$$
\begin{aligned}
{ }^{1} b=\left\{K_{B}, A_{P}, K_{P}, K_{P Q}, A_{Q}, K_{Q}, A_{R}, K_{R},\right. \\
\left.A_{S}, K_{S}, K_{C S}, s_{m}, K_{S T}, A_{T}, K_{T}, s_{I}, K_{I}\right\}
\end{aligned}
$$

and $n$ denotes the sample number within one generated heartbeat $\left(n=0,1,2, \ldots, N_{1}-1\right)$. Each heartbeat segment has a maximum length defined by its width parameters $\left(K_{x}\right)$. Thus, the sum of all segment lengths is $N_{1}=K_{B}+K_{P}+K_{P Q}+K_{Q}+K_{R}+K_{S}-K_{C S}+K_{S T}+K_{T}+K_{I}$.

\subsection{The PQRST complex with adjustable $Q$ and $S$ waves}

The modelling of the $\mathrm{Q}$ and $\mathrm{S}$ waves by their linear functions is the main enhancement on the previous model. Using linear functions instead of a Gaussian monopulse allows for the adjustment of the shape more precisely. All other modelling functions in this model are the same as those in the previous model, except for Equations (4) and (6).

Equation (4) is replaced by concatenation of two functions:

$Q_{1}(k)=-A_{Q} \frac{k}{K_{Q 1}} ;$ for $0 \leq k \leq K_{Q 1}$,

$Q_{2}(k)=A_{Q} \frac{k}{K_{Q 2}}-A_{Q} ;$ for $0 \leq k \leq K_{Q 2}$,

where $K_{Q_{1}}$ is the width of the downward deflection, $K_{Q_{2}}$ is the width of upward deflection, and $A_{Q}$ is the amplitude of the Q wave.

Equation (6) is replaced by a concatenation of two functions:

$S_{1}(k)=-A_{S} \frac{k}{K_{S 1}} ;$ for $0 \leq k \leq K_{S 1}$,

$S_{2}(k)=A_{S} \frac{k}{s_{S}}-A_{S} ;$ for $0 \leq k \leq K_{S 2}$,

where $K_{S 1}$ is the width of the downward deflection, and $K_{S 2}$ is the width of the upward deflection. Slope parameter $s_{s}$ represents the

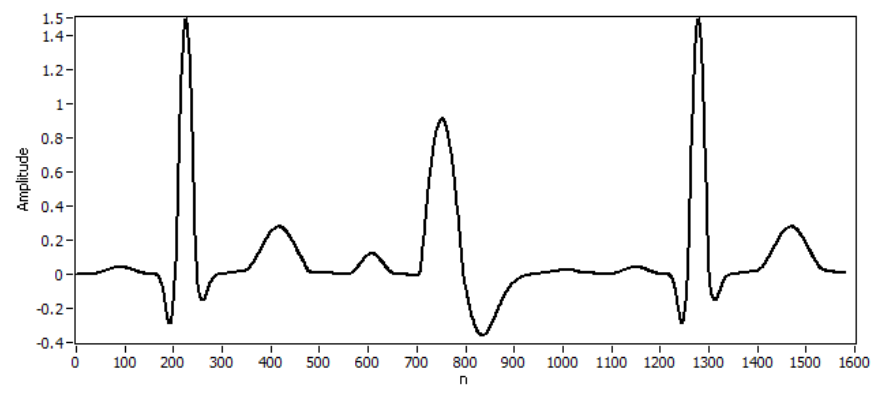

Figure 2. A modelled ECG containing a PVC heartbeat. upward deflection. Parameter $A_{S}$ is the amplitude of the $\mathrm{S}$ wave. The argument of $S\left(K_{S}-K_{C S}\right)$ in Equation (7) is replaced here by $K_{s 2}$.

A complete heartbeat $H\left({ }^{2} b, n\right)$ is then adjusted using the modified set of parameters ${ }^{2} b$ for the second variant:

$$
\begin{array}{r}
{ }^{2} b=\left\{K_{B}, A_{P}, K_{P}, K_{P Q}, A_{Q}, K_{Q 1}, K_{Q 2}, A_{R}, K_{R},\right. \\
\left.A_{S}, K_{S 1}, S_{S}, K_{S 2}, s_{m}, K_{S T}, A_{T}, K_{T}, S_{I}, K_{I}\right\} .
\end{array}
$$

Here, $n$ denotes the sample number $n=0,1,2, \ldots, N_{2}-1$, and $N_{2}=K_{B}+K_{P}+K_{P Q}+K_{Q 1}+K_{Q 2}+K_{R}+K_{S 1}+K_{S 2}+K_{S T}+K_{T}+K_{I}$ is the sum of all segment width parameters.

\subsection{Creating a custom ECG}

The custom ECG signal model is created by the following method. The function of one heartbeat is generated using any of the described variants as $H(b, n)$, where $b$ is the set of parameters ${ }^{1} b$ or ${ }^{2} b$ and $n=0,1,2, \ldots, N=N_{1}-1$ in the case of ${ }^{1} b$ or $N=N_{2}-1$ in the case of ${ }^{2} b$. A complete ECG signal can now be built by concatenating multiple heartbeats $H_{f}\left(b_{i}, n\right)$. The set of parameters $b_{i}$ can vary in each consecutive heartbeat or even continuously throughout a heartbeat. The beginning and end segment of each heartbeat aligns smoothly with the isoelectric line, so there are no artefacts present at the position of their joint.

The concatenation of the functions forming the sequence of the heartbeats may lead to unnatural sharp edges present in the resulting signal, so the generated heartbeat is filtered using a Savitzky-Golay smoothing filter with a window size of 7 :

$$
\begin{aligned}
H_{f}(b, n) & =\frac{1}{21}\{-2 H(b, n-3)+3 H(b, n-2)+ \\
& +6 H(b, n-1)+7 H(b, n)+6 H(b, n+1)+ \\
& +3 H(b, n+2)-2 H(b, n+3)\}
\end{aligned}
$$

for $n=0,1,2, \ldots, N$, where $H(b, n \pm j)=0$ if the sum $n \pm j$ is out of the specified interval for $n$. The filter plays a significant role while using the second model variant, because it forms the smooth shape of the Q and S waves.

This ECG model allows for the creation of a completely custom-defined ECG signal, which can include various irregular heartbeats present at custom positions. Even mixing the heartbeats generated from both the proposed model variants is possible. For example, a short signal containing a premature ventricular contraction (PVC) is modelled in Figure 2 using the first model variant.

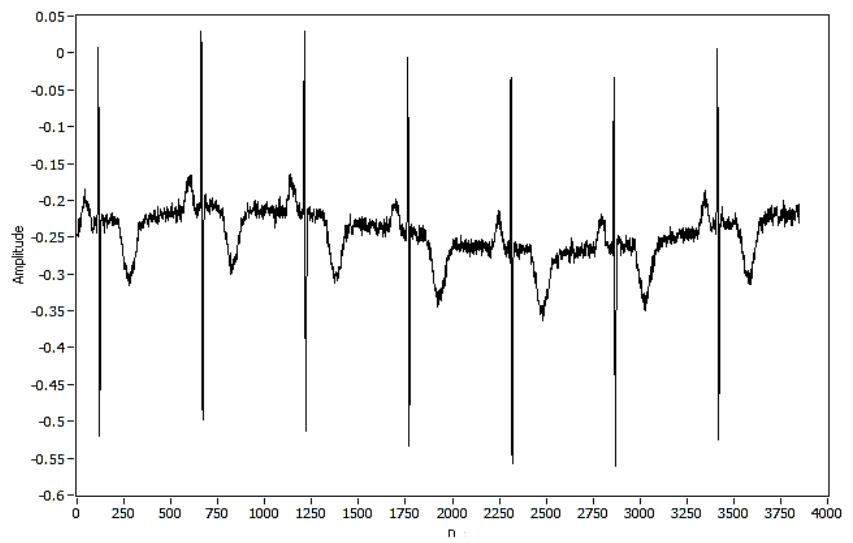

Figure 3. A modelled example of hypokalaemia ECG with noise and respiration added. 


\subsection{Modelling additive signals caused by respiration and external noise sources}

To make the modelled signal more realistic, different artefacts can be easily added to it. The respiration causes a baseline wander in the ECG signal. This means that the isoelectric line periodically changes its position, relatively slowly. A simple way of modelling this effect is to add a sinewave of the respiration frequency into the ECG signal, which may vary from about 0.2 $\mathrm{Hz}$ to $0.5 \mathrm{~Hz}$ (approximately 12 to 30 breaths per minute).

The interfering parasitic signal from external sources is modelled by the white noise and the inducted periodic signal from the powerline. If necessary, powerline noise can also be added in the form of a small sinewave with a powerline frequency of $50 \mathrm{~Hz}$ or $60 \mathrm{~Hz}$.

An example of a synthetized ECG signal in relation to hypokalaemia, with the addition of noise and the effect of respiration, can be seen in Figure 3 .

\section{AN EVALUATION OF THE PROPOSED ECG SIGNAL MODELS}

At the beginning of this study, the proposed models were evaluated using a database in the LabVIEW Biomedical Toolkit. The database represented the reference signals created from large-scale experimental data by medical experts. To test the possibilities of QRS pattern generation using the proposed model, its parameters were adjusted to fit typical irregular heartbeats. As a reference, irregular heartbeats associated with various diagnoses were generated using the ECG signal generator of the LabVIEW Biomedical Toolkit [27]. Each reference pattern $H_{r}(n)$ was extracted from a $60 \mathrm{bpm}$ signal, generated with a sampling frequency of $512 \mathrm{~Hz}$ and containing one QRS complex with a length of $1 \mathrm{~s}$. The set of model parameters $b$ was found using the Differential Evolution (DE) optimization algorithm [28] using the principle in [29]. The optimal mode
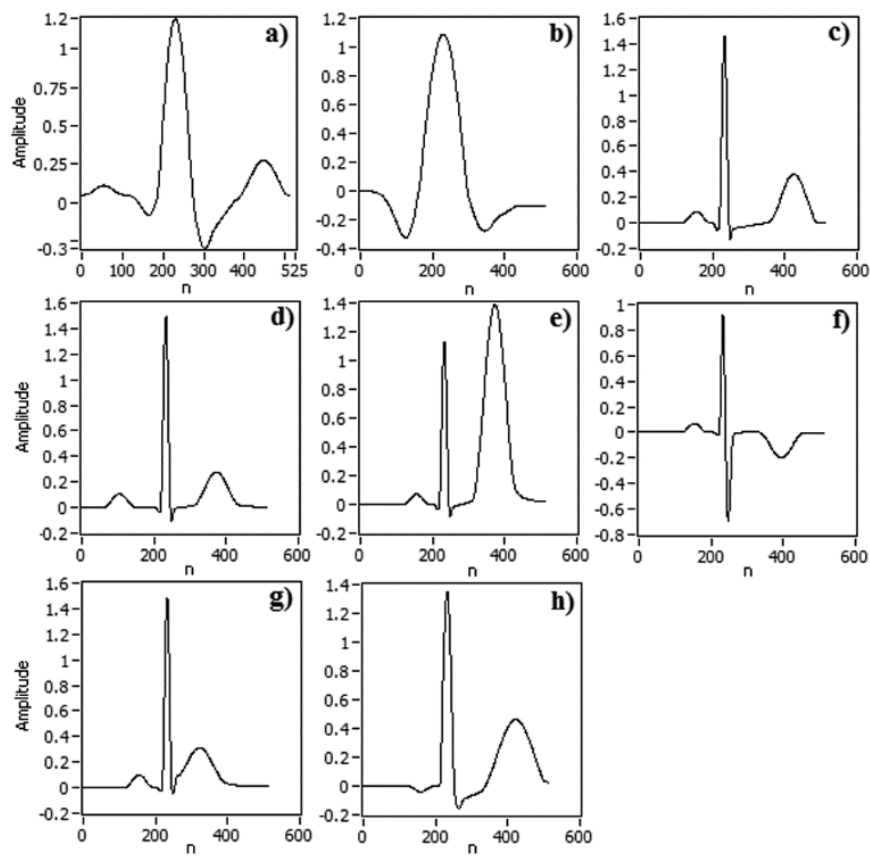

Figure 4. ECG patterns generated by the first model variant: a) atrial tachycardia; b) ventricular tachycardia; c) junctional tachycardia; d) atrioventricular block; e) hyperkalaemia; f) hypokalaemia; g) hypercalcaemia; h) hypocalcaemia.
Table 1. DE parameter bounds for the first and second model.

\begin{tabular}{ccc}
\hline $\mathbf{1}_{\mathbf{b}}$ & min & max \\
\hline$K_{B}$ & 0 & 130 \\
$A_{P}$ & -0.2 & 0.1 \\
$K_{P}$ & 10 & 100 \\
$K_{P Q}$ & 0 & 60 \\
$A_{Q}$ & 0 & 0.5 \\
$K_{Q}$ & 10 & 150 \\
$A_{R}$ & 1 & 2 \\
$K_{R}$ & 10 & 150 \\
$A_{S}$ & 0 & 1 \\
$K_{S}$ & 10 & 200 \\
$K_{C S}$ & -5 & 150 \\
$S_{m}$ & 1 & 150 \\
$K_{S T}$ & 0 & 110 \\
$A_{T}$ & -0.5 & 1 \\
$K_{T}$ & 50 & 200 \\
$S_{I}$ & 0 & 50 \\
\hline
\end{tabular}

\begin{tabular}{ccc}
\hline $\mathbf{2}_{\mathbf{b}}$ & min & max \\
\hline$K_{B}$ & 0 & 130 \\
$A_{P}$ & -0.2 & 0.1 \\
$K_{P}$ & 10 & 100 \\
$K_{P Q}$ & 0 & 60 \\
$A_{Q}$ & 0 & 0.5 \\
$K_{Q 1}$ & 0 & 70 \\
$K_{Q 2}$ & 0 & 50 \\
$A_{R}$ & 1 & 2 \\
$K_{R}$ & 10 & 150 \\
$A_{S}$ & 0 & 1 \\
$K_{S 1}$ & 0 & 50 \\
$S_{S}$ & 1 & 110 \\
$K_{S 2}$ & 0 & 50 \\
$S_{m}$ & 1 & 150 \\
$K_{S T}$ & 0 & 100 \\
$A_{T}$ & -0.5 & 1 \\
$K_{T}$ & 50 & 200 \\
$S_{I}$ & 0 & 150 \\
\hline
\end{tabular}

parameters for the best fit are found by means of the iterative minimization of each parameter $b$ :

$b=\arg \min _{b} P R D$

where $P R D$ is the normalized mean square difference between the reference heartbeat ECG signal and the modelled heartbeat ECG signal:

$P R D=\frac{\left\|H_{r}(n)-H_{f}(b, n)\right\|_{2}}{\left\|H_{r}(n)\right\|_{2}} \cdot 100 \%$.

In each iteration, the DE algorithm takes the reference ECG signal, compares it to the ECG generated based on the current parameter set $b$, and updates the parameter set, as summarized in Figure 4. This is repeated until a sufficiently low PRD or maximum iteration count is reached.

The initial bounds of the $b$ parameters were set in the following way: -0.2 to 2 for the amplitude parameters, $0-150$ for the segment length parameters, and 0-200 for the slope parameters. After the first few iterations of DE, the $b$ parameter bounds were adjusted according to the data shown in Table 1. The length parameter of the last segment $K_{I}$ is calculated so that the total sum of length parameters $N=N_{1}-1$ or $N=N_{2}-1$ is always equal to 512; thus, it is excluded from the set of parameters that are being optimized. The signal output of a model with such a configuration does not need resampling.

Artificial QRS patterns were used as a test reference, corresponding to (a) atrial tachycardia, (b) ventricular tachycardia, (c) junctional tachycardia, (d) atrioventricular block, (e) hyperkalaemia, (f) hypokalaemia, (g) hypercalcemia, and (h) hypocalcaemia. The resulting ECG waveforms are shown in Figure 5.

The corresponding parameters found by the DE optimization and the resultant PRD for the first model are listed in Table 2. For the second model, the identified parameters and PRD are listed in Table 3.

The results were obtained using a simulation in the LabVIEW programming environment. The DE optimization used the uniform crossover method, a population size of 500, and a maximum number of iterations of 200 for all the testing results provided. The PRD values listed in all the tables are given as an average of ten runs of the DE algorithm. 
The second evaluation method is based on a comparison between the modelled signal and the real signal taken from a database of real patients' signals. The evaluation of the real ECG patterns is similar to that which was described in the previous subsection. Here, the reference pattern is made by means of extraction of a single heartbeat from the beginning of the first channel of each Massachusetts Institute of Technology - Beth Israel Hospital (MIT-BIH) database record [17]. The database records use a sampling frequency of $360 \mathrm{~Hz}$. The reference pattern $H_{r}(n)$ is obtained by the resampling for a duration of $1 \mathrm{~s}$ at a sampling frequency of $512 \mathrm{~Hz}$. The $\mathrm{DE}$ algorithm remains the same as listed in Table 1 for the previous experiments. The modelling accuracy, evaluated by PRD, was assessed for the real ECG patterns taken from the MIT-BIH database and is presented in Table 4.

As evidenced in Table 4, each model is suitable for different types of patterns. The average PRD is better for the second model variant, although it should be noted that the extracted heartbeats possibly contain noise and artefacts that were not included in the signal model during this test. Noise causes a higher PRD even if there is a good fit between the original and modelled signal [30].

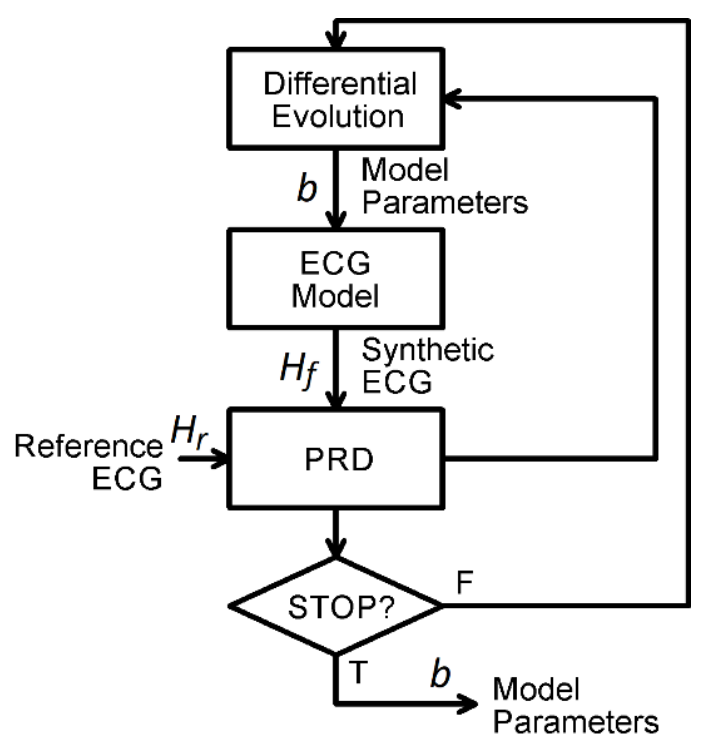

Figure 5. An ECG fitting algorithm for finding the set model parameters.

Table 2. The first variant model parameter sets for each pattern.

\begin{tabular}{|c|c|c|c|c|c|c|c|c|}
\hline \multirow{2}{*}{${ }^{1} b$} & \multicolumn{8}{|c|}{ Diagnosis } \\
\hline & $\mathbf{a}$ & b & c & d & e & $f$ & g & $\mathbf{h}$ \\
\hline$K_{B}$ & 10 & 0 & 117 & 61 & 121 & 121 & 117 & 124 \\
\hline$A_{p}$ & 0.03 & 0 & 0.0045 & 0.053 & 0.035 & 0.035 & 0.05 & -0.02 \\
\hline$K_{P}$ & 93 & 23 & 79 & 91 & 73 & 69 & 79 & 75 \\
\hline$K_{P Q}$ & 0 & 0 & 0 & 48 & 6 & 13 & 5 & 0 \\
\hline$A_{Q}$ & 0.135 & 0.325 & 0.065 & 0.04 & 0.04 & 0.02 & 0.03 & 0 \\
\hline$K_{a}$ & 85 & 140 & 25 & 21 & 21 & 22 & 20 & 15 \\
\hline$A_{R}$ & 1.15 & 1.09 & 1.52 & 1.55 & 1.17 & 1 & 1.55 & 1.37 \\
\hline$K_{R}$ & 84 & 133 & 23 & 23 & 23 & 15 & 22 & 36 \\
\hline As & 0.35 & 0.28 & 0.16 & 0.13 & 0.11 & 0.75 & 0.6 & 0.16 \\
\hline$K_{s}$ & 114 & 182 & 15 & 15 & 15 & 26 & 14 & 54 \\
\hline$K_{c s}$ & 61 & 100 & 5 & 2 & 4 & -3 & 5 & 27 \\
\hline$S_{m}$ & 61 & 119 & 96 & 17 & 26 & 35 & 1 & 87 \\
\hline$K_{S T}$ & 52 & 57 & 101 & 52 & 56 & 64 & 6 & 42 \\
\hline$A_{T}$ & 0.13 & 0 & 0.19 & 0.132 & 0.685 & -0.1 & 0.115 & 0.225 \\
\hline$K_{T}$ & 127 & 77 & 126 & 116 & 112 & 112 & 116 & 184 \\
\hline$s_{l}$ & 0 & 0 & 2 & 9 & 9 & 7 & 10 & 19 \\
\hline$K_{I}$ & 8 & 0 & 31 & 87 & 89 & 67 & 138 & 9 \\
\hline$P R D$ in \% & 7,8 & 7,61 & 11,12 & 11,3 & 14,0 & 9,89 & 13,4 & 7,65 \\
\hline
\end{tabular}

Table 3. The second variant model parameter sets for each pattern.

\begin{tabular}{|c|c|c|c|c|c|c|c|c|}
\hline \multirow{2}{*}{${ }^{2} b$} & \multicolumn{8}{|c|}{ Diagnosis } \\
\hline & a & b & c & d & e & $f$ & g & $h$ \\
\hline$K_{B}$ & 10 & 0 & 117 & 61 & 121 & 121 & 117 & 124 \\
\hline$A_{P}$ & 0.03 & 0 & 0.0045 & 0.053 & 0.035 & 0.035 & 0.05 & -0.02 \\
\hline$K_{p}$ & 93 & 23 & 79 & 91 & 73 & 69 & 79 & 75 \\
\hline$K_{P Q}$ & 1 & 1 & 14 & 50 & 13 & 43 & 14 & 17 \\
\hline$A_{Q}$ & 0.13 & 0.33 & 0.065 & 0.065 & 0.047 & 0 & 0.06 & 0 \\
\hline$K_{Q 1}$ & 55 & 55 & 12 & 12 & 12 & 0 & 11 & 0 \\
\hline$K_{Q 2}$ & 31 & 37 & 7 & 7 & 7 & 0 & 7 & 0 \\
\hline$A_{R}$ & 1.15 & 1.09 & 1.52 & 1.55 & 1.17 & 1 & 1.55 & 1.37 \\
\hline$K_{R}$ & 77 & 137 & 22 & 22 & 22 & 12 & 23 & 32 \\
\hline$A_{s}$ & 0.38 & 0.27 & 0.18 & 0.16 & 0.12 & 0.48 & 0.1 & 0.19 \\
\hline$K_{S 1}$ & 32 & 44 & 9 & 7 & 7 & 12 & 5 & 15 \\
\hline$s_{s}$ & 62 & 108 & 4 & 4 & 6 & 6 & 2 & 8 \\
\hline$K_{S 2}$ & 33 & 31 & 4 & 4 & 6 & 7 & 4 & 4 \\
\hline$s_{m}$ & 53 & 97 & 138 & 64 & 14 & 1 & 29 & 55 \\
\hline$K_{S T}$ & 52 & 52 & 100 & 56 & 53 & 74 & 1 & 62 \\
\hline$A_{T}$ & 0.12 & 0 & 0.2 & 0.13 & 0.665 & -0.1 & 0.12 & 0.23 \\
\hline$K_{T}$ & 119 & 87 & 137 & 126 & 126 & 126 & 133 & 183 \\
\hline$s_{l}$ & 17 & 0 & 0 & 128 & 32 & 0 & 23 & 0 \\
\hline$K_{I}$ & 9 & 45 & 11 & 76 & 72 & 48 & 118 & 0 \\
\hline$P R D$ in $\%$ & 9,5 & 10,6 & 9,18 & 9,59 & 6,69 & 13,9 & 10,6 & 11,4 \\
\hline
\end{tabular}




\section{CONCLUSIONS}

Two basic ECG signal models have been presented in this paper based on a set of basic function parameters. The models allow for the description of irregularieties in the shapes of the characteristic waves in one heartbeat. Irregularities in the

Table 4. The DE parameter bounds corresponding to common diagnoses: the second model variant.

\begin{tabular}{|c|c|c|}
\hline \multirow[t]{2}{*}{ Record No. } & \multicolumn{2}{|c|}{$P R D$ in $\%$} \\
\hline & Method 1 & Method 2 \\
\hline 100 & 16.23 & 15.09 \\
\hline 101 & 15.36 & 15.16 \\
\hline 102 & 31.09 & 31.61 \\
\hline 103 & 12.13 & 21.79 \\
\hline 104 & 38.73 & 40.89 \\
\hline 105 & 19.37 & 15.11 \\
\hline 106 & 18.83 & 19.41 \\
\hline 107 & 31.37 & 19.33 \\
\hline 108 & 32.25 & 21.88 \\
\hline 109 & 16.19 & 10.85 \\
\hline 111 & 34.42 & 33.86 \\
\hline 112 & 8.56 & 7.66 \\
\hline 113 & 24.91 & 23.36 \\
\hline 114 & 18.11 & 39.85 \\
\hline 115 & 12.99 & 13.19 \\
\hline 116 & 6.77 & 13.29 \\
\hline 117 & 7.02 & 19.22 \\
\hline 118 & 11.17 & 10.49 \\
\hline 119 & 5.73 & 6.13 \\
\hline 121 & 11.19 & 12.4 \\
\hline 122 & 5.88 & 4.87 \\
\hline 123 & 7.19 & 6.79 \\
\hline 124 & 15.37 & 10.53 \\
\hline 200 & 21.11 & 20.89 \\
\hline 201 & 14.56 & 14.35 \\
\hline 202 & 20.01 & 41.62 \\
\hline 203 & 26.68 & 22.96 \\
\hline 205 & 8.73 & 8.81 \\
\hline 207 & 41.01 & 44.82 \\
\hline 208 & 42.65 & 24.79 \\
\hline 209 & 11.03 & 9.84 \\
\hline 210 & 34.98 & 24.75 \\
\hline 212 & 31.71 & 48.62 \\
\hline 213 & 17.41 & 16.71 \\
\hline 214 & 36.57 & 29.05 \\
\hline 215 & 11.71 & 9.09 \\
\hline 217 & 33.06 & 14.69 \\
\hline 219 & 19.02 & 16.11 \\
\hline 220 & 5.93 & 7.46 \\
\hline 221 & 16.25 & 15.58 \\
\hline 222 & 24.72 & 27.21 \\
\hline 223 & 15.84 & 14.59 \\
\hline 228 & 20.91 & 19.39 \\
\hline 230 & 15.69 & 11.59 \\
\hline 231 & 17.57 & 12.39 \\
\hline 232 & 20.18 & 22.15 \\
\hline 233 & 35.43 & 28.58 \\
\hline 234 & 21.88 & 19.43 \\
\hline Average & 20.11 & 19.54 \\
\hline
\end{tabular}

consecutive heartbeats can be modelled by combining both models. In addition, the respiratory and external electromagnetic interferences can be included in the models. The model parameters were identified from real ECG records using the differential evolution optimization strategy. The normalized mean square difference PRD was the parameter describing the modelling accuracy. Besides the physically acquired ECG signals, the modelling accuracy was evaluated by the ECG signals generated by the LabVIEW Biomedical Toolkit and real ECG records from MIT-BIH.

The ECG model evaluation using signals from databases showed that modelling the low-frequency $\mathrm{Q}$ and $\mathrm{S}$ waves is better in the first model. The second ECG model, with linearly modelled segments $\mathrm{Q}$ and $\mathrm{S}$, is more suitable in the case of steeper waveforms. A lower number of model parameters is the advantage of the first model, also an advantage in the DE optimization. The relatively complicated adjustment of the heart rate of long-term signals is another drawback of the second variant presented. Resampling the acquired ECG signal to a suitable rate is the easiest way of suppressing this disadvantage for both model variants.

\section{ACKNOWLEDGEMENT}

The work is a part of the project supported by the Science Grant Agency of the Slovak Republic (Project No. 1/0722/18).

\section{REFERENCES}

[1] M. Crescentini, M. Marchesi, A. Romani, M. Tartagni, P. A. Traverso, Bandwidth limits in Hall effect-based current sensors, ACTA IMEKO 6 (2017) 4, 17-24. http://dx.doi.org/10.21014/acta imeko.v6i4.478

[2] L. Klaus, B. Arendacká, M. Kobusch, T. Bruns, Dynamic torque calibration by means of model parameter identification, ACTA IMEKO 4 (2015) 2, 39-44.

http://dx.doi.org/10.21014/acta imeko.v4i2.211

[3] Y. Yamakawa, T. Yamazaki, Mathematical model of checkweigher with electromagnetic force balance system, ACTA IMEKO 3 (2014) 2, 9-13.

http://dx.doi.org/10.21014/acta imeko.v3i2.86

[4] R. Xiao-ping, J. Wang, W. Ombati, Y. Wang, B. Chen, Ch. Cai, Surface analytical model and sorption artifact designing method, ACTA IMEKO 5 (2016) 3, 76-80. http://dx.doi.org/10.21014/acta imeko.v5i3.299

[5] M. L. D'Angelo, F. Cannella, M. D'Imperio, M. Bianchi, A preliminary approach to study the behavior of human fingertip at contact via experimental test and numerical model, ACTA IMEKO 6 (2017) 2, 81-88.

http://dx.doi.org/10.21014/acta imeko.v6i2.393

[6] Y. Hiranaka, S. Miura, T. Taketa, Hybrid backward simulator for determining causal heater state with resolution improvement of measured temperature data through model conformation, ACTA IMEKO 6 (2017) 1, 13-19.

http://dx.doi.org/10.21014/acta imeko.v6i1.390

[7] M. Lazzaroni, M. Citterio, S. Latorre, A. Lanza, P. Cova, N. Delmonte, F. Giuliani, Thermal modeling and characterization for designing reliable power converters for LHC power supplies, ACTA IMEKO 3 (2014) 4, 17-25. http://dx.doi.org/10.21014/acta imeko.v3i4.147

[8] L. Sujbert, G. Orosz, Frequency domain identification of data loss models, ACTA IMEKO 6 (2017) 4, 61-67. http://dx.doi.org/10.21014/acta imeko.v6i4.476

[9] L. Y. Taha, E. Abdel-Raheem, EEG signal extraction utilizing null space approach, Proc. of the 19th IEEE International Symposium on Signal Processing and Information Technology, Dec. 10 - 12, 
2019, Ajman, United Arab Emirates, 2019, 1-5. http://dx.doi.org/10.1109/ISSPIT47144.2019.9001818

[10] C. Lin, J. Jhang, Cardiac arrhythmia signal modeling and recognizing based on fractional low order estimation, Proc. of the 9th IEEE International Conference on System Engineering and Technology, ICSET 2019, Oct. 7, 2019, Shah Alam, Malaysia. Art. no. 8906421, 370-373. http://dx.doi.org/10.1109/ICSEngT.2019.8906421

[11] P. Dolinský, I. Andráš, L. Michaeli, J. Šaliga, High Sensitivity Experimetal QRS Detector, $28^{\text {th }}$ IEEE International conference Radioelektronika, Apr. 19 - 20, 2018, Prague, Czechia, 1-4, ISBN 978-1-5386-2485-2.

[12] E. Balestrieri, L. De Vito, F. Picariello, I. Tudosa, A novel method for compressed sensing based sampling of ECG signals in medical-IoT era, Proc. of the MeMeAAt Conference, 2019, Istanbul, Turkey. http://dx.doi.org/10.1109/MeMeA.2019.8802184

[13] E. Balestrieri, P. Daponte, L. De Vito, F. Picariello, S. Rapuano, I. Tudosa, Experimental assessment of a novel CS-based acquisition method for ECG signals in IoMT, Proc. of the 24th IMEKO TC4 International Symposium, Sept. 17-20, 2019, Xi'An, China, ISBN: 978-606-13-5238-8.

[14] I. Andráš, P. Dolinský, L. Michaeli, J. Šaliga， Sparse signal acquisition via compressed sensing and principal component analysis, Measurement Science Review, Ústav merania SAV 18(5), 2018, 175-182, ISSN 1335-8871.

[15] A. Affanni, Dual-channel electrodermal activity and an ECG wearable sensor for measuring mental stress from the hands, Acta IMEKO 8 (2019) 1, 56-63. http://dx.doi.org/10.21014/acta imeko.v8i1.562

[16] M. Feli, F. Abdali-Mohammadi, 12 lead electrocardiography signals compression by a new genetic programming based mathematical modeling algorithm, Biomedical Signal Processing and Control 54 (2019) 101596. http://dx.doi.org/10.1016/i.bspc.2019.101596

[17] A. L. Goldberger, L. A. N. Amaral, L. Glass, J. M. Hausdorff, P. Ch. Ivanov, R. G. Mark, J. E. Mietus, G. B. Moody, C. K. Peng, H. E. Stanley, PhysioBank, PhysioToolkit, and PhysioNet: components of a new research resource for complex physiologic signals, Circulation 101(23) (2000) e215-e220.

[18] J. Richardson, L. J. Haywood, V. K. Murthy, G. Harvey, A mathematical model for ECG wave forms and power spectra, Mathematical Biosciences 12(3-4) (1971), 321-328.

[19] V. Chutchavong, K. Nualon, O. Sangaroon, K. Janchitrapongvej, A mathematical model for ECG waveform using rational Bézier curves and Bernstein polynomials, Proc. of the 11th International Conference on Electrical Engineering/Electronics, Computer, Telecommunications and Information Technology (ECTI-CON), 2014, Nakhon Ratchasima, 1-5.
[20] H. Alquran, A. M. Alqudah, I. A. Qasmieh, S. Almashaqbeh, Gaussian model of electrooculogram signals, Proc. of the IEEE Jordan International Joint Conference on Electrical Engineering and Information Technology, JEEIT, Apr. 9 - 11, 2019, Amman, Jordan. art. no. 8717499, 293-296. http://dx.doi.org/10.1109/JEEIT.2019.8717499

[21] J. Paulo do Vale Madeiro, J. A. L. Marques, T. Han, R. C. Pedros, Evaluation of mathematical models for QRS feature extraction and QRS morphology classification in ECG signals, Measurement: Journal of the International Measurement Confederation, 156 art. no. 107580 (2020). http://dx.doi.org/10.1016/i.measurement.2020.107580

[22] P. Kovacs, C. Bock, C. T. Dozsa, J. Meier, M. Huemer, Waveform Modeling by Adaptive Weighted Hermite Functions, 44th IEEE International Conference on Acoustics, Speech and Signal Processing (ICASSP), May 12 - 17, 2019, Brighton, United Kingdom, 1-23.

[23] F. Yang, B. Chen, K. Zhu, Z. Chu, Research on the fitting method for QRS wave group of ECG signals based on Ant Colony algorithm, Mechanisms and Machine Science 75 (2020), 1041 1049

http://dx.doi.org/10.1007/978-3-030-27053-7 89

[24] S. Javed, N. A. Ahmad, Mathematical modeling of real time ECG waveform, Science and Information Conference (SAI), July 10 12, 2018, London, United Kingdom, 606-614.

[25] M. Shyshkin, O. Butova, L. Fetiukhina, O. Akhiiezer, O. Dunaievska, K. Kyrychenko, Simulation of the ECG signal based on the analysis of the spectrum of its standard components, Proc. of the $2^{\text {nd }}$ IEEE Ukraine Conference on Electrical and Computer Engineering, UKRCON, July 2 - 6, 2019, Lviv, Ukraine, Art. no. 8879838, 229-233.

[26] E. J. Berbari, Principles of Electrocardiography, Biomedical Engineering Handbook: Second Edition, CRC Press, Danvers, USA, 2000. ISBN 0-8493-0461-X.

[27] National Instruments, LabVIEW Biomedical Toolbox, 2019. Austin, Texas, USA. Retrieved 19.6.2020 from: https://sine.ni.com/nips/cds/view/p/lang/de/nid/211023

[28] I. Andráš, P. Dolinský, L. Michaeli, J. Šaliga, A time domain reconstruction method of randomly sampled frequency sparse signal, Measurement 127 (2018), 68-77. https://doi.org/10.1016/j.measurement.2018.05.065

[29] I. Andráš, J. Šaliga, L. Michaeli, Compressed sensing with modelbased reconstruction, Proc. of the 24th IMEKO TC4 International Symposium, Sept. 17-20, 2019, Xi'An, China, ISBN: 978-606-13-5238-8.

[30] P. Dolinský, I. Andráš, L. Michaeli, J. Šaliga, A new simple ECG signal model, Proc. of the 24th IMEKO TC4 International Symposium, Sept. 17-20, 2019, Xi'An, China, ISBN: 978-606-13$5238-8$. 